\title{
Selection of endoscopic or transcranial surgery for tuberculum sellae meningiomas according to specific anatomical features: a retrospective multicenter analysis (KOSEN-002)
}

\author{
*Doo-Sik Kong, MD, PhD, ${ }^{1}$ Chang-Ki Hong, MD, PhD, ${ }^{2}$ Sang Duk Hong, MD, MS, ${ }^{3}$ \\ Do-Hyun Nam, MD, PhD, ${ }^{1}$ Jung-II Lee, MD, PhD, ${ }^{1}$ Ho Jun Seol, MD, PhD, ${ }^{1}$ Jiwoong Oh, MD, ${ }^{2}$ \\ Dong Gyu Kim, MD, PhD, ${ }^{4}$ and Yong Hwy Kim, MD, PhD ${ }^{4}$
}

\begin{abstract}
Departments of ${ }^{1}$ Neurosurgery and ${ }^{3}$ Otorhinolaryngology-Head and Neck Surgery, Samsung Medical Center, Sungkyunkwan University; ${ }^{2}$ Department of Neurosurgery, Gangnam Severance Hospital, Yonsei University; and ${ }^{4}$ Department of Neurosurgery, Seoul National University Hospital, Seoul National University College of Medicine, Seoul, Korea
\end{abstract}

\begin{abstract}
OBJECTIVE The endoscopic endonasal approach (EEA) and the transcranial approach (TCA) are good options for the treatment of tuberculum sellae (TS) meningiomas. The objective of this study was to identify the key anatomical features in TS meningiomas and compare the two surgical approaches.
\end{abstract}

METHODS The authors retrospectively reviewed clinical data in 178 patients with TS meningiomas treated at 3 institutions between January 2010 and July 2016. Patients with tumors encasing the internal carotid artery or anterior cerebral artery or involving the anterior clinoid process or cavernous sinus were excluded. Tumors were classified as high-lying or low-lying based on their location, and involvement of the optic canal was evaluated. The surgical outcomes of EEA and TCA were analyzed according to the relevant anatomical features.

RESULTS During the study period, 84 patients underwent EEA and 94 patients underwent TCA. Based on preoperative MR images, 43 (24.2\%) meningiomas were classified as high-lying tumors, $126(70.8 \%)$ as low-lying, and $9(5.0 \%)$ as nonspecific. Gross-total resection (GTR) was performed in 145 patients (81.5\%); the GTR rate did not differ significantly between the EEA and TCA groups. Of 157 patients with preoperative visual disturbance, 140 had improved or stable vision postoperatively. However, 17 patients (9.6\%) experienced some visual deterioration after surgery. The TCA group had a worse visual outcome than the EEA group in patients with preoperative optic canal involvement $(77.6 \%$ vs $93.2 \%$, $p=0.019$ ), whereas there was no significant difference in visual outcome based on whether tumors were high-lying or low-lying.

CONCLUSIONS The results of this study support EEA over TCA, at least with respect to visual improvement with acceptable complications, although TCA is still an effective approach for TS meningioma.

https://thejns.org/doi/abs/10.3171/2017.11.JNS171337

KEYWORDS tuberculum sellae; meningioma; approach; transcranial; endoscope; visual outcome; pituitary surgery

$\mathrm{T}$ He tuberculum sellae (TS) is a bony ridge that forms the anterior aspect of the sella. ${ }^{8}$ Meningiomas arising from the TS and planum sphenoidale (PS) account for approximately $15 \%$ of WHO grade I meningiomas. ${ }^{1,12,16,18,26}$ Initially, patients with these lesions often present with visual disturbances caused by compression of the optic apparatus. Therefore, the primary goal of treatment for TS meningiomas is visual restoration as well as gross-total resection (GTR).

Advanced microsurgical techniques have contributed to reducing morbidity and improving surgical management of meningiomas arising from the TS. With recent ad-

ABBREVIATIONS EEA = endoscopic endonasal approach; GTR = gross-total resection; KOSEN = Korean Society of Endoscopic Neurosurgery; MPR = multiplanar reconstruction; $\mathrm{PS}=$ planum sphenoidale; $\mathrm{TCA}$ = transcranial approach; $\mathrm{TS}=$ tuberculum sellae; VIS = visual impairment score.

SUBMITTED July 12, 2017. ACCEPTED November 14, 2017.

INCLUDE WHEN CITING Published online May 18, 2018; DOI: 10.3171/2017.11.JNS171337.

* D.S.K. and C.K.H. contributed equally to this work. 
vances in high-definition endoscopic techniques, the endoscopic endonasal approach (EEA) allows direct access to the TS and opens a new corridor to the subchiasmatic space compared with the transcranial approach (TCA). 3,6 , 15,17,29,31 No consensus has been reached on which surgical approach is more advantageous for TS meningiomas without radiographic evidence of vascular encasement in terms of optimal GTR, visual outcome, and morbidity. In addition, the optimal indication for EEA in the treatment of TS meningiomas has not been identified.

To address this issue, we focused on visual outcomes and characterized specific anatomical features with respect to the sphenoid sinus, sella, and optic canal in a cohort of patients with TS meningiomas who underwent the TCA or the EEA. We hypothesized that the EEA might have advantages over the TCA under specific conditions, for instance, tumors with pneumosinus dilatans (high-lying tumors), deep extension into the sellar area (low-lying tumors), or extensions into the optic canal. The objective of this study was to identify the preoperative anatomical characteristics to be considered when selecting the appropriate approach and to clarify the optimal indications for using EEA for TS meningiomas. Therefore, we measured tumor height and size, planum height, depth of tumor involvement in the sella, and optic canal involvement on preoperative high-resolution MRI and investigated the prognostic factors to affect GTR and visual outcome.

\section{Methods}

This study was approved by the institutional review boards at 3 institutions (Samsung Medical Center, Gangnam Severance Hospital, and Seoul National University Hospital). We retrospectively reviewed imaging data and clinical records in cases involving patients who were diagnosed with TS meningioma and surgically treated at the 3 institutions between January 2010 and July 2016. Medical records and pre- and postoperative MRI data, including thin-sectioned axial, coronal, and sagittal T1-weighted imaging with contrast, were reviewed. Inclusion criteria for this study were the following: 1) TS or PS meningioma confirmed by radiological and histopathological examinations; 2) available pre- and postoperative MRI data; 3 ) available results of visual examinations, such as visual acuity and field assessment, from both pre- and postoperative assessments; and 4) no previous surgery for the same meningioma. To reduce selection bias, we excluded patients with the following conditions: 1) a tumor completely encasing the internal carotid artery or anterior cerebral artery complex; 2) a tumor extending laterally to the optic nerve; 3 ) having undergone previous surgical treatment for the same tumor; or 4) a tumor involving the anterior clinoid process, cavernous sinus, petrous apex, sphenoidal ridge, or olfactory groove. We identified 178 patients who were treated at the 3 institutions during the study period and met these criteria. Postoperatively, all patients underwent MRI with gadolinium enhancement 3 months after surgery and at regular 1-year intervals. The extent of resection was determined by an independent radiologist from each institution. The visual acuity and visual field, assessed with a Humphrey field analyzer, were evaluat- ed both preoperatively and 3 to 6 months after surgery. Some patient data were used in authors' previous studies for analysis with respect to reconstruction techniques and visual results. ${ }^{25,29}$

\section{Preoperative Morphological Analysis With MRI}

A blinded investigator retrospectively inspected preoperative, coronal Gd-enhanced T1-weighted images and T2-weighted images obtained with a standard 1.5- or 3.0T MRI system. 3D high-resolution MRI provided reliable information to define the exact relationships between the optic nerve and tumors involving the optic canal. Multiplanar reconstruction (MPR) imaging allows for a more precise identification of optic canal involvement, encasement of the anterior cerebral artery complex, and the relationship of the surrounding anatomical structures. In 141 of 178 patients, high-resolution contrast-enhanced MPR MRI was performed preoperatively to identify complex anatomical structures. To characterize the anatomical variability of the lesions, we evaluated anatomical features of the tumor with respect to the relationship to the sphenoid sinus, sella, and optic nerve (Fig. 1). Specifically, we measured tumor size and height as well as the depth of tumor involvement in the sella, planum height, and the presence of optic canal involvement. Depending on the degree of pneumatization of the ethmoid or sphenoid sinus, the planum height was defined as the distance from the frontobasal line (line from the anterior to posterior aspect of cribriform plate) to the top of the planum (Fig. 1). When the planum height was greater than $5 \mathrm{~mm}$, the tumor was classified as high-lying and required more brain retraction during surgery (Figs. 2B and 3). The sella depth was defined as the length from the TS to the deepest portion of the tumor within the sella. When the depth of the sella was greater than $5 \mathrm{~mm}$, the tumor was classified as low-lying (Figs. 2C and 4). The presence of optic canal involvement was compared with the asymmetricity of vision as well as the preoperative MRI (Figs. 2D and 5).

\section{Evaluation of Visual Function}

Patients underwent visual acuity and visual field examinations (Humphrey 24-2 protocol, Humphrey Field Analyzer II, Zeiss Humphrey Systems, Carl Zeiss Ophthalmic Systems Inc.). An ophthalmological evaluation based on the guidelines of the German Ophthalmological Society was performed by an ophthalmologist preoperatively and 1 week, 3 to 6 months, and 1 year after surgery. Visual function was quantified by the visual impairment score (VIS), which was the sum of visual acuity and visual field defect scores, to allow for exact comparisons. ${ }^{9,20,28}$ The postoperative visual field and visual acuity assessments were repeated 1 week, 3 to 6 months, and 1 year after surgery. Chi-square or Fisher's exact tests were used to compare categorical variables, and a Kruskal-Wallis test was performed to compare the visual outcome according to the surgical approaches.

\section{Selection of Approach}

In this retrospective study, the TCA surgeries were performed by 4 surgeons (C.H., J.L., H.S., and D.N.) who have 


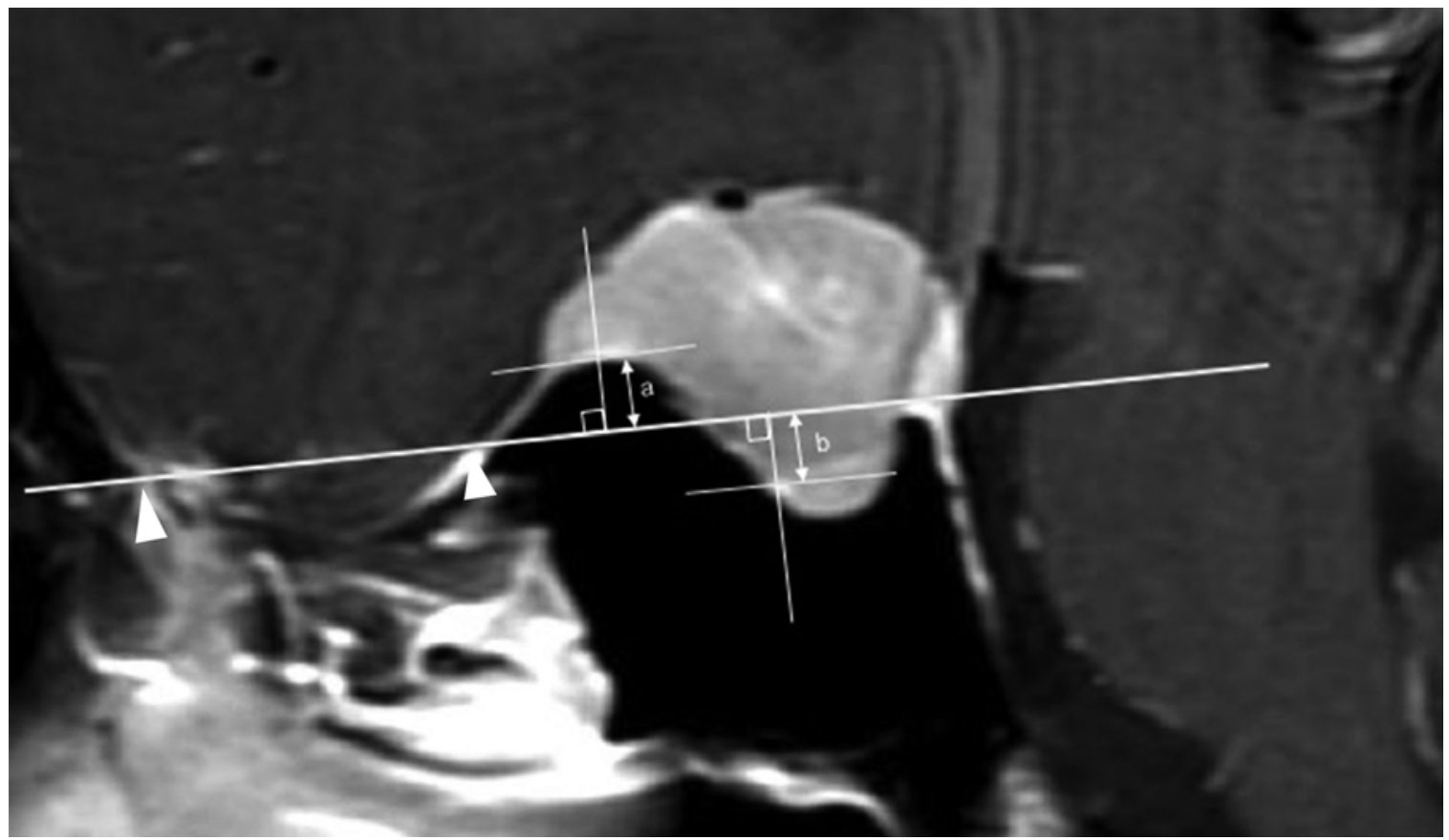

FIG. 1. Morphological measurement of TS meningioma on a sagittal T1-weighted MR image. The planum height (a) is defined as the distance from the frontobasal line (line passing through the anterior [white arrowhead, left] and posterior [white arrowhead, right] ends of the cribriform plate) to the top of the planum. The sella depth (b) is defined as the length from the TS to the deepest portion of the tumor within the sella.

specialized in transcranial brain tumor surgery for over 10 years. The TCA procedures included subfrontal, frontobasal interhemispheric, and supraorbital approaches. Approach selection was based on the surgeon's preference and the tumor shape. The EEA surgeries were performed by 3 surgeons (D.S.K., Y.K., and D.N.). Age, sex, operation date, follow-up period, surgical approach, surgeon of record, preoperative clinical manifestation, postoperative changes in vision, and postoperative complications were investigated retrospectively. Gross-total resection (GTR) was defined as $100 \%$ tumor removal in the operation record and no remaining tumor lesion on postoperative MRI according to an independent radiologist. Near-total resection was defined as greater than $90 \%$ tumor removal in the operation record and visible lesion remaining on postoperative MR images despite absence of visible mass in the surgical field.

\section{EEA Procedure}

The surgical procedure for EEA was similar among institutions. The patient's head was held in place with Mayfield pin fixation, and neuronavigational guidance was used. The nasal cavity was packed with cottonoid patties soaked in 1:10,000 epinephrine before draping the field, and the middle turbinate and septal mucosa were subsequently infiltrated with 1:100,000 epinephrine to reduce bleeding from the nasal mucosa. We emphasize the importance of this step because, in most cases, the main cause of a blurred surgical field is not tumor bleeding but blood from the adjacent nasal mucosa. In our experience, the covering of the mucosa could be changed with a new epinephrine-soaked cottonoid patty coat every 2 hours, considering the effective duration of epinephrine. A nasoseptal pedicled flap was harvested in advance. A minimal posterior septectomy followed by wide sphenoidotomy was performed for each case. The boundary of the bony opening included the sellar floor, TS, and PS. Laterally, the bilateral medial opticocarotid recess was the lateral margin. The bone overlying the medial opticocarotid recess was carefully removed with a diamond drill with copious irrigation. Vertical linear incision into the dura was performed after bipolar coagulation of the dura covering the superior intercavernous sinus and tumor base. Accordingly, the inferior surface of the tumor was simultaneously exposed when the dura was opened, because TS meningiomas arise from this dural portion. After adequate internal debulking of the tumor with suction or an ultrasonic aspirator, the tumor capsule could be sharply dissected from the adjacent arachnoid membrane. Cauterization of the dural base prior to opening the dura could reduce tumor bleeding during the internal debulking of the tumor. If the tumor volume was small, an EEA could provide safe resection without interrupting the chiasmatic cistern. However, large tumors often interfere with the chiasmatic cistern and encase vascular structures, such as the H-complex of anterior cerebral arteries. In such cases, careful consideration was required to avoid inadvertent injury to the vascular structures. Finally, the involved medial optic canal was opened, and the tumor involving the optic canal was removed, in cases in which preoperative MRI showed definite invasion or when tumors were visible inside the optic canal under intraoperative angled endoscopic inspection. When the optic canal was opened, a 


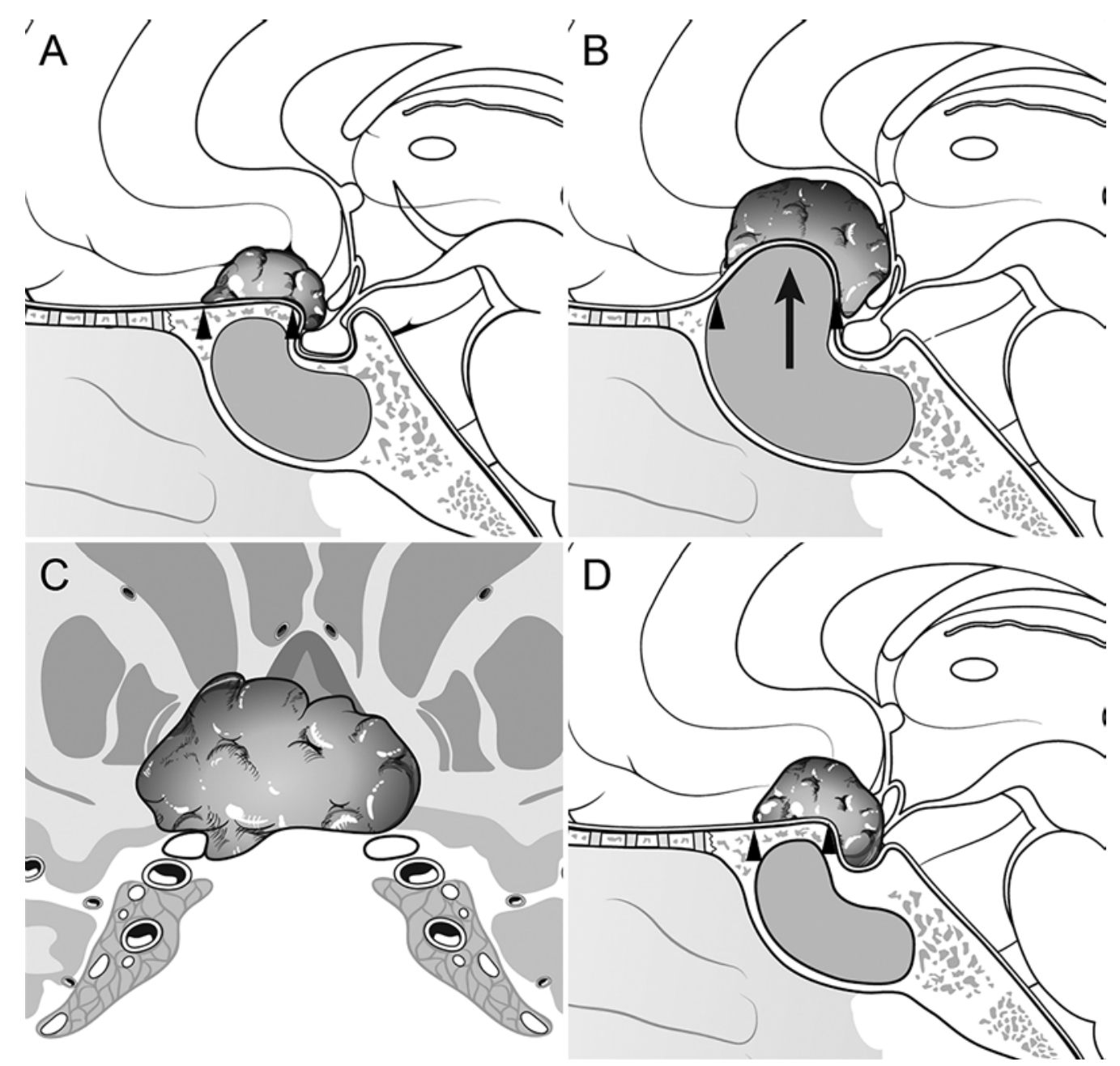

FIG. 2. Schematic drawing of TS meningioma. Meningioma usually arises from the dura over the TS and extends to the PS and sellar fossa. A: Typical shape of TS meningioma. B: High-lying tumor with pneumosinus dilatans. High-lying tumors were defined in this study as meningiomas with pneumosinus dilatans $>5 \mathrm{~mm}$ above the frontobasal line. C: Tumor involving the optic canal on the right side. D: Low-lying tumor extending into the sella $>5 \mathrm{~mm}$ below the tuberculum. The black arrowheads indicate the anterior and posterior points of cribriform plate. Copyright Yong Hwy Kim. Published with permission.

sickle knife and microscissors were carefully used to prevent injury to the ophthalmic artery. After the tumor was removed, the fascia lata graft was laid down with a bilayer (inlay and onlay) button. Fascia was placed intradurally as an inlay graft and extradurally as an onlay graft. Finally, the harvested nasoseptal flap was rotated into position to reconstruct the skull base defect. Postoperative CSF lumbar drainage was performed for 3 to 5 days.

\section{TCA Procedure}

In the 94 patients treated with TCA, access to the TS was achieved through 64 unilateral pterional approaches, 5 supraorbital approaches, and 25 bilateral subfrontal approaches. The direction of surgical approach was chosen according to the location of the tumor, height of the tumor, asymmetry of blurred vision, and surgeons' preference. The dura was opened in a curvilinear fashion, and tumor was exposed at the TS. After devascularization using bipolar cautery along the floor of the planum and tuberculum, the tumor was debulked with careful microdissection to avoid interruption of the arachnoid membrane as possible. Anterior clinoidectomy or optic canal decompression was not always performed. In the TCA, the falciform ligament was first opened, and then the intracanalicular portion of the tumor at the entrance of the canal was removed. If tumor still remained, the optic canal was completely unroofed with extreme caution. The decision whether to unroof the optic canal was based on the surgeon's judgment. We found that unroofing the optic canal as well as the release of the falciform ligament was performed in 26 of 94 patients who underwent TCA.

\section{Results}

For this study, 178 patients who fulfilled the inclusion criteria for resection of a TS meningioma and underwent either EEA $(n=84)$ or TCA $(n=94)$ between January 2010 and July 2016 were identified. The patients included 42 men and 136 women. Their mean age was 53.8 years (range 31-79 years), and the mean follow-up period was 


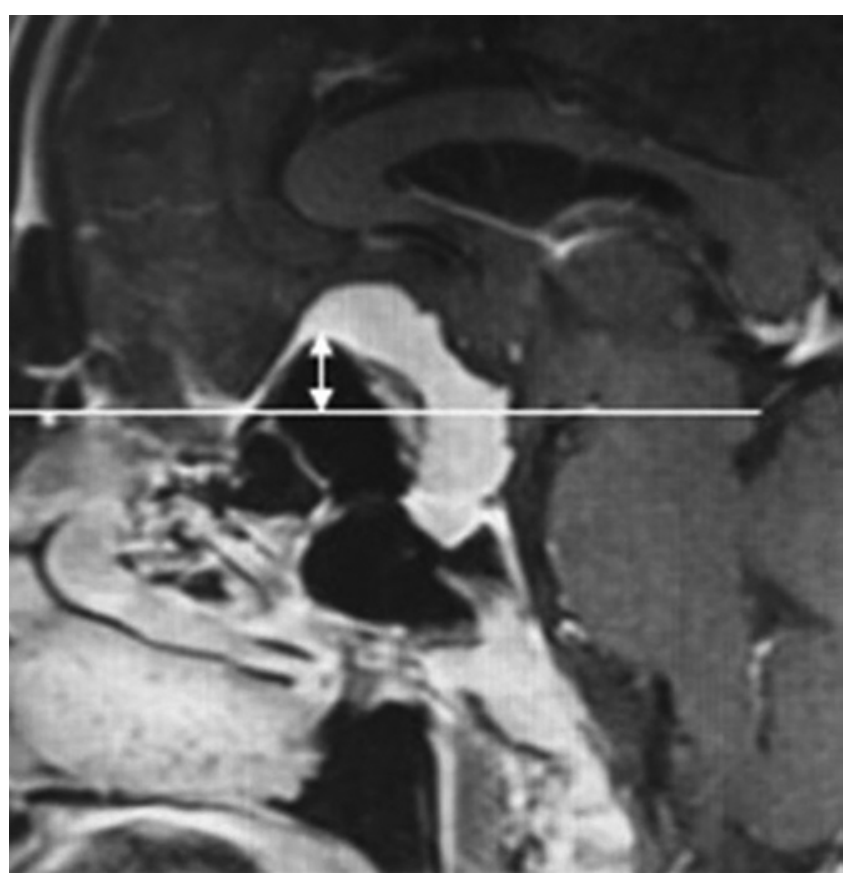

FIG. 3. Midsagittal Gd-enhanced T1-weighted MR image obtained in a 34-year-old woman who presented with the visual disturbance. The image shows a tumor of the high-lying category, defined as TS meningiomas with pneumosinus dilatans $>5 \mathrm{~mm}$ above the frontobasal line (measurement indicated by double-headed arrow), which may require more cerebral retraction during surgery.
28.0 months (range 3.1-70.6 months). In this study, 157 of the 178 patients $(88.2 \%)$ initially presented with visual disturbance, and 21 patients (11.8\%) were asymptomatic. The mean value of the maximum tumor diameter was 2.2 $\mathrm{cm}$ (range $0.8-4.9 \mathrm{~cm}$ ). Most patients had a histologically determined WHO grade I meningioma without adjacent brain invasion, but 9 patients had WHO grade II or III meningiomas. Pathological diagnosis included meningothelial, transitional, and fibrous types. Based on preoperative MR images and according to our classification, 43 (24.2\%) tumors were high-lying meningiomas, $126(70.8 \%)$ were low-lying tumors, and 9 (5.0\%) were nonspecific with respect to high or low location. The demographic characteristics were summarized in Table 1.

GTR was performed in 145 of 178 patients (81.5\%); 33 patients (18.5\%) underwent subtotal resection of the tumor. The major causes of incomplete resection included thick adhesion to the surrounding vascular structures or optic apparatus, remaining tumor tissue within the optic canal, and hard calcified tumor mass. Of the 157 patients with preoperative visual disturbance, 111 patients $(70.7 \%)$ had improved vision, and 29 (18.5\%) patients had stable function without impairment. However, 17 patients (10.8\%) experienced some deterioration after surgery. Overall, the mean VIS score improved from $45.2 \pm 26.0$ to $23.6 \pm$ 24.3 after surgery. The surgical outcomes of the 2 surgical groups are summarized in Table 2.

\section{Comparison of Overall Data for the EEA and TCA Groups Extent of Resection}

The mean preoperative tumor size did not differ sig-
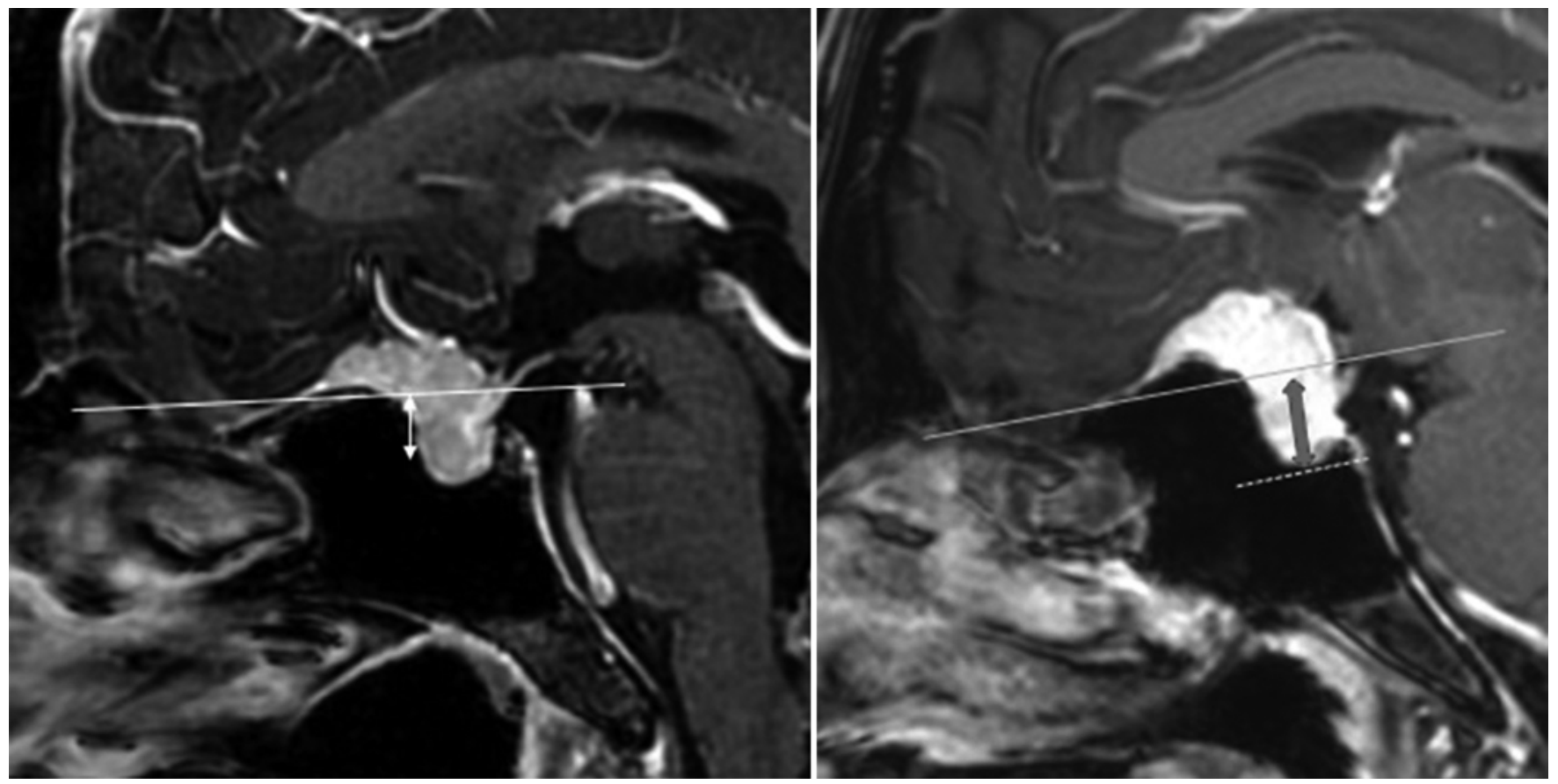

FIG. 4. Left: Midsagittal Gd-enhanced T1-weighted MR image obtained in a 64-year-old woman demonstrating a low-lying TS meningioma extending into the sella $>5 \mathrm{~mm}$ below the tuberculum (double-headed arrow indicates $5 \mathrm{~mm}$, for reference). Right: Midsagittal Gd-enhanced T1-weighted MR image obtained in a 57-year-old woman showing another example of a low-lying TS meningioma. The double-headed arrow indicates the length of $5 \mathrm{~mm}$. 

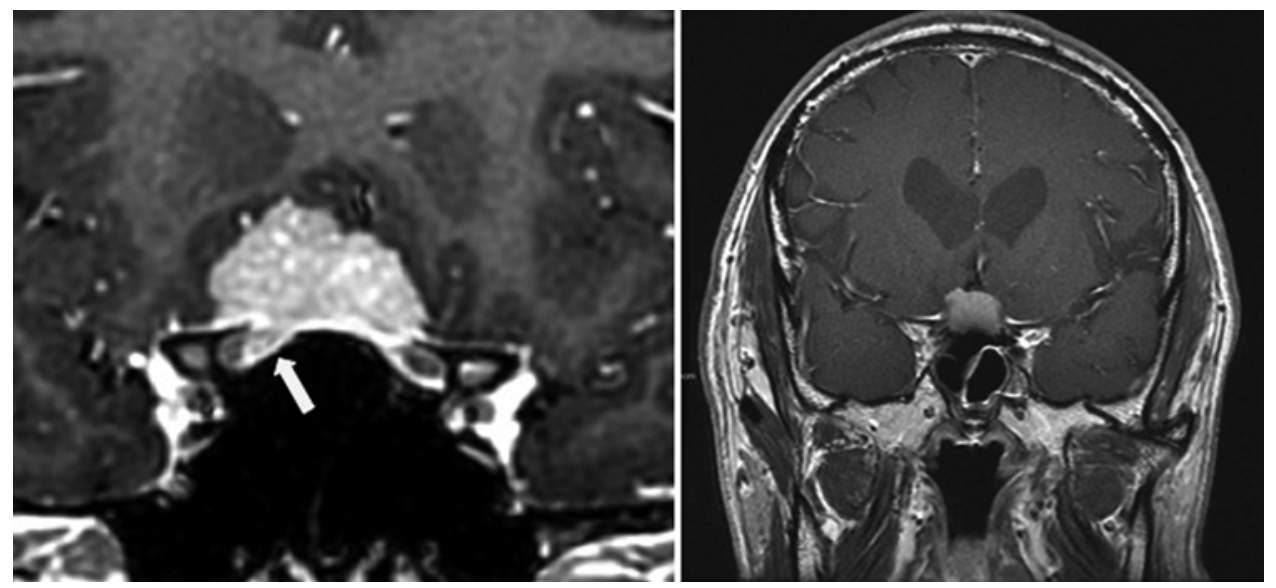

FIG. 5. Left: Coronal view of 3D multiplanar MR image obtained in a 43-year-old woman showing a meningioma involving the optic canal on the right side (arrow). Right: Coronal Gd-enhanced T1-weighted MR image obtained in a 36-year-old woman showing a tumor without optic canal involvement.

nificantly between the EEA group and the TCA group (2.4 $\pm 0.7 \mathrm{~cm}$ vs $2.1 \pm 0.8 \mathrm{~cm}, \mathrm{p}>0.05)$, and there was no statistically significant between-groups difference in the proportion of patients in whom GTR was achieved $(83.3 \%$ vs $79.8 \%, \mathrm{p}=0.651)$.

TABLE 1. Clinical and demographic characteristics of 178 patients with TS meningioma

\begin{tabular}{|c|c|c|c|}
\hline Characteristic & $\operatorname{EEA}(n=84)$ & $\mathrm{TCA}(n=94)$ & $p$ Value \\
\hline \multicolumn{4}{|l|}{ Sex } \\
\hline Female & 64 & 72 & NS \\
\hline Male & 20 & 22 & \\
\hline Mean age (yrs) & $54.2 \pm 13.6$ & $53.7 \pm 11.0$ & NS \\
\hline \multicolumn{4}{|l|}{ Initial presentation } \\
\hline Visual disturbance & 80 & 77 & NS \\
\hline Headache & 1 & 2 & \\
\hline Dizziness & 1 & 2 & \\
\hline Incidental & 2 & 13 & \\
\hline Mean preop VIS & $45.9 \pm 25.9$ & $45.0 \pm 21.7$ & NS \\
\hline \multicolumn{4}{|l|}{ Features of tumor } \\
\hline Mean size $(\mathrm{cm})$ & $2.4 \pm 0.7$ & $2.1 \pm 0.8$ & NS \\
\hline Optic canal involvement & 60 & 51 & 0.013 \\
\hline \multicolumn{4}{|l|}{ Types of tumors } \\
\hline High-lying tumor & 23 & 20 & NS \\
\hline Low-lying tumor & 56 & 70 & NS \\
\hline Not specific & 5 & 4 & \\
\hline \multicolumn{4}{|l|}{ Institution } \\
\hline SMC & 26 & 49 & \\
\hline SNU & 56 & 6 & \\
\hline GSH & 2 & 39 & \\
\hline
\end{tabular}

GSH = Gangnam Severance Hospital; NS = not significant; SMC = Samsung Medical Center; SNU = Seoul National University Hospital.

Values are numbers of cases unless otherwise indicated. Mean values are shown with standard deviations.

\section{Visual Outcomes}

Visual outcomes are essential for evaluating the outcomes of TS meningioma surgery because of the risk of injury to the optic apparatus. A greater proportion of patients with preoperative visual disturbance showed improvement in their visual symptoms after the EEA than after the TCA $(85.0 \%$ vs $55.8 \%, \mathrm{p}<0.001)$. In addition, fewer patients in the EEA group experienced postoperative visual deterioration than in the TCA group (5.0\% vs $17.1 \%, \mathrm{p}=0.015)$.

\section{Postoperative Complications}

The main complications after the EEA were CSF leakassociated complications (10 of 84 patients, 11.9\%; 6 patients had postoperative meningitis and 4 patients required reconstruction surgery for CSF leaks. Other complications included an abscess in 1 patient and cardiac death in 1 patient. The TCA group had cerebral retraction-associated complications, including stroke in 1 patient, intracerebral hematoma in 1 patient, and subdural hematoma in 2 patients. Other complications included abscess, meningitis, and death of unknown cause. Although only $4.8 \%$ of patients in the EEA group underwent reconstruction surgery, EEA still had limitations for reconstruction when compared with the TCA group. In contrast, the TCA group had higher rates of complications associated with cerebral retraction. In this study, we excluded minor complications, such as nasal crusting or anosmia after EEA and pain or numbness at the craniotomy site or temporal wasting after TCA (Table 1). Because olfactory groove meningioma was excluded from this study, there was no patient who complained of preoperative anosmia, and no new anosmia were observed postoperatively.

\section{Specific Anatomical Features Affecting Visual Outcomes} High-Lying Tumor (with pneumosinus dilatans)

Since Benjamins first described in 1918 that enlargement of the sphenoid sinus, called "pneumosinus dilatans," was associated with changes secondary to PS meningiomas, ${ }^{11}$ this sphenoid blistering has often been found in 
TABLE 2. Comparison of surgical outcomes in the EEA and TCA groups

\begin{tabular}{|c|c|c|c|}
\hline Characteristic & EEA & TCA & p Value \\
\hline \multicolumn{4}{|l|}{$\operatorname{GTR}(n=178)$} \\
\hline Overall (\%) & $70 / 84(83.3 \%)$ & $75 / 94(79.8 \%)$ & \multirow{3}{*}{ NS } \\
\hline High-lying (43) & $17 / 23(73.9 \%)$ & $16 / 20(80 \%)$ & \\
\hline Low-lying (126) & $42 / 56(75 \%)$ & $59 / 70(84.3 \%)$ & \\
\hline \multicolumn{4}{|l|}{ Visual outcome ( $n=157)$} \\
\hline \multicolumn{4}{|l|}{ Overall } \\
\hline Improved (\%) & $68(85)$ & $43(55.8)$ & $<0.001$ \\
\hline No change (\%) & $8(10)$ & $21(27.3)$ & \\
\hline Deteriorated (\%) & $4(5)$ & $13(16.9)$ & 0.015 \\
\hline \multicolumn{4}{|l|}{ High-lying (n = 38) } \\
\hline Improved or no change (\%) & $22(100)$ & $16(100)$ & NS \\
\hline Deteriorated $(\%)$ & $0(0)$ & $0(0)$ & \\
\hline \multicolumn{4}{|l|}{ Low-lying ( $n=114$ ) } \\
\hline Improved or no change (\%) & $50(92.6)$ & $48(80)$ & \multirow{2}{*}{0.063} \\
\hline Deteriorated $(\%)$ & $4(7.4)$ & $12(20)$ & \\
\hline \multicolumn{4}{|l|}{ Optic canal involvement $(n=108)$} \\
\hline Improved or no change (\%) & $55(93.2)$ & $38(77.6)$ & \multirow{2}{*}{0.019} \\
\hline Deteriorated $(\%)$ & $4(6.8)$ & $11(22.4)$ & \\
\hline \multicolumn{4}{|l|}{ Complications } \\
\hline Overall & 10 & 6 & \\
\hline Meningitis & 6 & 1 & \\
\hline CSF leaks & 4 & & \\
\hline Abscess & 1 & 1 & \\
\hline Stroke & & 1 & \\
\hline $\mathrm{ICH}$ or $\mathrm{SDH}$ & & 2 & \\
\hline Death of unknown cause & 1 & 1 & \\
\hline
\end{tabular}

$\mathrm{ICH}=$ intracerebral hematoma; $\mathrm{SDH}=$ subdural hematoma.

TS meningiomas. In this study, we investigated the incidence of TS meningiomas accompanied by pneumosinus dilatans, defined as high-lying tumors. We hypothesized that high-lying tumors resulting from abnormal enlargement of the sphenoid sinus might provide some difficulties in reaching the tumor with an EEA or require more cerebral retraction with a TCA. We defined pneumosinus dilatans as a planum height $>5 \mathrm{~mm}$ above the frontobasal line (from the anterior to posterior aspect of the cribriform plate). By assessing preoperative MR images, we found that 38 of 157 patients (24.2\%) with preoperative visual disturbance had a high-lying tumor. All patients with a high-lying meningioma demonstrated improvement or no change of visual outcome after EEA or TCA. We found visual outcomes did not differ significantly between EEA and TCA groups even in the cases with the planum height $>5 \mathrm{~mm}(\mathrm{p}>0.05)$. In summary, these results indicate that a high-lying tumor may not significantly affect the selection of the surgical approach for TS meningiomas.

\section{Low-Lying Tumor}

We defined low-lying tumors as tumors extending downward from the sella $>5 \mathrm{~mm}$. The sella depth was defined as the length from the TS to the deepest portion of the tumor within the sella. We hypothesized that EEA might have relative advantages over the TCA in terms of resection rate or visual outcomes for low-lying tumors. In this series, 114 of 157 patients with preoperative visual disturbance (72.6\%) had tumor extending over $5 \mathrm{~mm}$ into the sella. Of these patients with low-lying tumor and preoperative visual disturbance, 54 underwent EEA and 60 underwent TCA. Of those who underwent EEA, 50 (90\%) had improved visual outcome or showed no change of visual status postoperatively, whereas in the group treated with TCA, 58 patients $(80 \%)$ showed improved or stable visual outcomes. In particular, the visual deterioration rate was higher in patients who underwent TCA than in those whose tumors were managed by EEA ( $20 \%$ vs $7.4 \%$ ). We found that patients with low-lying tumors tended to have better visual outcomes after EEA than after TCA, but the difference was not statistically significant $(\mathrm{p}=0.063)$.

\section{Optic Canal Involvement}

3D high-resolution MPR imaging can be used to identify optic canal involvement. Preoperative MRI showed that unilateral or bilateral optic canal invasion occurred in 108 patients (60.7\%). Preoperative MRI evaluation of optic canal invasion was consistent with the clinical manifestation of asymmetrical preoperative visual impairment. According to our classification, the preoperative images 
showed 43 (24.2\%) high-lying meningiomas, 126 (70.8\%) low-lying tumors, and $9(5.0 \%)$ nonspecific tumors. Patients with optic canal involvement-38 patients (77.6\%) in the TCA group and 55 patients $(93.2 \%)$ in the EEA group-exhibited visual improvement or no change in visual status after surgery; this between-groups difference was statistically significant $(\mathrm{p}=0.019$, OR $3.98,95 \%$ CI 1.18-13.44). In contrast, of the 49 patients without optic canal involvement, none of the 21 patients who underwent EEA and 2 of 28 patients who underwent TCA had worse visual outcome after surgery and there was no statistically significant difference in outcome $(\mathrm{p}>0.05)$ (Table 2$)$.

\section{Discussion}

The tuberculum sellae (TS) is the indentation between the anterior aspect of the sella turcica and the posterior declining part of the planum sphenoidale (PS). ${ }^{8}$ Depending on the specific anatomical characteristics of the tumor, such as size, shape, and optic canal involvement, the optimal approach for tumor resection should be adjusted. ${ }^{10,18,22,27,30}$ The EEA can be a good alternative to treat TS meningiomas, as they are typically on the midline without eccentric lateral growth. A lower surgical route such as an EEA provides a wider and panoramic view, early identification of the tumor, effective decompression of optic pathway with less manipulating the optic pathway and brain, clear identification of the superior hypophyseal artery under the optic nerves as well as improved visualization of the medial optic canal. ${ }^{3}$ In contrast, an upper route such as a TCA provides several corridors to access the tumor with few restrictions on tumor size, extent, or vascular encasement. ${ }^{30}$ In addition, increased surgical freedom with the TCA allows improved delicate microdissection and enables surgeons to perform GTR. To date, the definitive choice of upper or lower route for TS meningioma remains controversial. ${ }^{3,7}$ Since TS meningiomas arise from the chiasm area, they commonly present with visual impairment, even in small tumors. In this setting, special attention should be paid to carefully decompress the optic pathway and completely preserve the blood vessels supplying the optic pathway. ${ }^{14,24,30}$ As a result, visual outcomes and complications are important issues for distinguishing these 2 approaches in TS meningiomas. ${ }^{3}$

\section{Selection of an Approach Based on Specific Imaging Features}

We focused on specific anatomical features including, the sphenoid sinus, sella, and optic canal, with regard to visual outcome. Assessment of the surgical relevance of various types of tumors is essential. The extent of the lesion and surrounding anatomical structures can influence the selection of approaches, such as EEA or TCA. We identified certain anatomical variations among TS meningiomas. We initially hypothesized that each approach has advantages for tumors with specific anatomical features, such as pneumosinus dilatans, deep extension into the sella or optic canal involvement. Hirst et al. suggested that neuroradiological findings of an enlarged sphenoid sinus without hyperostosis of the sphenoid bone in patients with visual disturbance were highly suggestive of a meningioma involv- ing the optic canal. ${ }^{11}$ We initially evaluated which approach might be more appropriate for this type of tumor. A tumor accompanied by pneumosinus dilatans, which we referred to as a high-lying tumor, may require more vigorous brain retraction via the conventional pterional or subfrontal TCA. To assess the degree of cerebral retraction, the size, consistency of the tumor, and surrounding tumor edema as well as the height of the planum must be comprehensively considered. To reduce the confounding variables, we included only the height of planum, excluding the tumor factor itself in this study. However, we found that even high-lying tumors were removed without difficulty via both EEA and TCA as assessed by GTR and visual outcomes. The next consideration was the TS angle (angle from the frontobasal line to the sella). As suggested by de Notaris et al., ${ }^{8}$ a wide suprasellar corridor would allow an easier route for EEA. Based on these findings, we hypothesized that the shape of the TS meningioma could be determined based on the angle of the TS. For instance, TS meningiomas with a wide angle between the PS and TS tend to extend deep into the sella turcica and the lower route may be more appropriate. Our results showed that the EEA was more advantageous in preserving vision for tumors extending into the sella, but a significant difference was not shown. This result seems reasonable because the lower route could improve visualization of low-lying tumors. We expect that additional data will strengthen this hypothesis.

Finally, we found that for patients with tumors involving the optic canal, there was a significant difference in visual outcome between the EEA and TCA groups. Intracranial release of the optic canal via the transcranial route is a challenging task because the tumor usually compresses the optic nerve from the inferomedial side to the superolateral side. In the optic pathway, optic nerves are usually displaced superolaterally at the chiasmatic cistern, but the entrance of the optic canal does not have space for displacement because the anterior clinoid process and optic strut surrounds the optic canal. As a result, optic nerve around the entrance of optic canal (as viewed from the intracranial side) is subjected to be substantial compression and can be severely kinked. Accordingly, it is most important to decompress the entrance to the optic canal. In addition, even a small amount of tumor invasion into the optic canal leads to severe compression of the optic nerve due to the tight space of the optic canal in nature. In light of these concerns, endoscopic medial decompression of the optic canal can be a good alternative. A direct view of the optic canal and subsequent drilling of the TS is a useful adjunct to enable safe and complete tumor resection without a blind spot even for tumors involving bilateral optic canals. ${ }^{2,4,10,21,24,30}$ In the literature, resection of TS meningiomas has been associated with an $8 \%$ to $42 \%$ risk of postoperative visual impairment as a result of optic nerve manipulation or devascularization by vasospasm or manual coagulation. . $^{2,5,9,13,16,19,23,24,26,32}$ Less optic nerve manipulation and possibly preserving the perforating artery to the optic apparatus are important for preserving vision and preventing undesired visual impairment. This study has some limitations since it was designed as a retrospective and nonrandomized study. To overcome these limitations, we collected data from 3 institutions for the 
same time period. In addition, there was a high possibility of selecting relatively low-risk tumors, such as tumors without vascular encasement or extended involvement of neighboring anatomical structures in the EEA group. To avoid inherent selection bias, we excluded potentially high-risk tumors, such as clinoidal, sphenocavernous, and petroclival meningiomas, from both groups. A multicenter study of surgical outcome may have a problem with downward leveling of surgical technique and outcome from the difference between individual institutes. A similar number of surgeons participating in this study in both groups could compensate for this issue. Despite the superiority of EEA over TCA for TS meningioma, EEA still had a major weakness, compared with the TCA, with respect to the significantly higher risk of CSF leaks. To address this troublesome complication of EEA, a variety of surgical techniques, such as the button-layer and gasket-sealing techniques, have been developed in recent years. However, we suggest that the most important points of these surgical techniques may be ease of use, the short learning curve, and expansible dexterity.

\section{Conclusions}

The results of this study support EEA over TCA, at least with respect to visual improvement with acceptable complications. The EEA may be good choice for treating TS meningiomas with discrete involvement of the optic canal or low-lying tumors. If the tumor involves surrounding structures other than the TS or encases a major vascular complex, the TCA is still an effective approach. To determine the optimal approach, high-resolution 3D MPR MRI will be useful for identifying tumor shape and optic canal involvement.

\section{Acknowledgments}

We would like to thank Dong-Su Jang, MFA, (medical illustrator, Medical Research Support Section, Yonsei University College of Medicine, Seoul, Korea) for his help with the illustrations.

This study was supported by a grant (HI16C-1111-020016 to Y. H. Kim) from the Korea Health Industry Development Institute (KHIDI), funded by the Ministry of Health \& Welfare, Republic of Korea; a grant (NRF-2017R1A2B2008412 to Y. H. Kim) from National Research Foundation of Korea by the Ministry of Science, ICT and Future Planning (MSIP) of Korea; and grants (NRF2015M3A9A7029740, NRF-2015M3C9A1044522, and NRF2015M3A9B5053642) of the National Research Foundation funded by MSIP of Korea; and by a Samsung Medical Center grant.

\section{References}

1. Arai H, Sato K, Okuda, Miyajima M, Hishii M, Nakanishi H, et al: Transcranial transsphenoidal approach for tuberculum sellae meningiomas. Acta Neurochir (Wien) 142:751-757, 2000

2. Attia M, Kandasamy J, Jakimovski D, Bedrosian J, Alimi M, Lee DL, et al: The importance and timing of optic canal exploration and decompression during endoscopic endonasal resection of tuberculum sella and planum sphenoidale meningiomas. Neurosurgery 71 (1 Suppl Operative):58-67, 2012

3. Bander ED, Singh H, Ogilvie CB, Cusic RC, Pisapia DJ, Tsiouris AJ, et al: Endoscopic endonasal versus transcranial approach to tuberculum sellae and planum sphenoidale meningiomas in a similar cohort of patients. J Neurosurg 128:40-48, 2018
4. Bassiouni H, Asgari S, Stolke D: Tuberculum sellae meningiomas: functional outcome in a consecutive series treated microsurgically. Surg Neurol 66:37-45, 2006

5. Chicani CF, Miller NR: Visual outcome in surgically treated suprasellar meningiomas. J Neuroophthalmol 23:3-10, 2003

6. Clark AJ, Jahangiri A, Garcia RM, George JR, Sughrue ME, McDermott MW, et al: Endoscopic surgery for tuberculum sellae meningiomas: a systematic review and meta-analysis. Neurosurg Rev 36:349-359, 2013

7. de Divitiis E, Esposito F, Cappabianca P, Cavallo LM, de Divitiis O: Tuberculum sellae meningiomas: high route or low route? A series of 51 consecutive cases. Neurosurgery 62:556-563, 2008

8. de Notaris M, Solari D, Cavallo LM, D’Enza AI, Enseñat J, Berenguer J, et al: The "suprasellar notch," or the tuberculum sellae as seen from below: definition, features, and clinical implications from an endoscopic endonasal perspective. J Neurosurg 116:622-629, 2012

9. Fahlbusch R, Schott W: Pterional surgery of meningiomas of the tuberculum sellae and planum sphenoidale: surgical results with special consideration of ophthalmological and endocrinological outcomes. J Neurosurg 96:235-243, 2002

10. Hayhurst C, Teo C: Tuberculum sella meningioma. Otolaryngol Clin North Am 44:953-963, viii-ix, 2011

11. Hirst LW, Miller NR, Hodges FJ III, Corbett JJ, Thompson S: Sphenoid pneumosinus dilatans. A sign of meningioma originating in the optic canal. Neuroradiology 22:207-210, 1982

12. Kane AJ, Sughrue ME, Rutkowski MJ, Shangari G, Fang $\mathrm{S}, \mathrm{McDermott} \mathrm{MW}$, et al: Anatomic location is a risk factor for atypical and malignant meningiomas. Cancer 117:12721278,2011

13. Lee JH, Jeun SS, Evans J, Kosmorsky G: Surgical management of clinoidal meningiomas. Neurosurgery 48:10121021, 2001

14. Li-Hua C, Ling C, Li-Xu L: Microsurgical management of tuberculum sellae meningiomas by the frontolateral approach: surgical technique and visual outcome. Clin Neurol Neurosurg 113:39-47, 2011

15. Linsler S, Fischer G, Skliarenko V, Stadie A, Oertel J: Endoscopic assisted supraorbital keyhole approach or endoscopic endonasal approach in cases of tuberculum sellae meningioma: which surgical route should be favored? World Neurosurg 104:601-611, 2017

16. Mahmoud M, Nader R, Al-Mefty O: Optic canal involvement in tuberculum sellae meningiomas: influence on approach, recurrence, and visual recovery. Neurosurgery 67:ons108ons119, 2010

17. Makarenko S, Carreras EM, Akagami R: Craniotomy for perisellar meningiomas: comparison of simple (appropriate for endoscopic approach) versus complex anatomy and surgical outcomes. J Neurosurg 126:1191-1200, 2017

18. Margalit NS, Lesser JB, Moche J, Sen C: Meningiomas involving the optic nerve: technical aspects and outcomes for a series of 50 patients. Neurosurgery 53:523-533, 2003

19. Mathiesen T, Kihlström L: Visual outcome of tuberculum sellae meningiomas after extradural optic nerve decompression. Neurosurgery 59:570-576, 2006

20. Morisako H, Goto T, Goto H, Bohoun CA, Tamrakar S, Ohata K: Aggressive surgery based on an anatomical subclassification of craniopharyngiomas. Neurosurg Focus 41(6):E10, 2016

21. Nanda A, Konar SK, Maiti TK, Bir SC, Guthikonda B: Stratification of predictive factors to assess resectability and surgical outcome in clinoidal meningioma. Clin Neurol Neurosurg 142:31-37, 2016

22. Nozaki K, Kikuta K, Takagi Y, Mineharu Y, Takahashi JA, Hashimoto N: Effect of early optic canal unroofing on the outcome of visual functions in surgery for meningiomas of 
the tuberculum sellae and planum sphenoidale. Neurosurgery 62:839-846, 2008

23. Pamir MN, Ozduman K, Belirgen M, Kilic T, Ozek MM: Outcome determinants of pterional surgery for tuberculum sellae meningiomas. Acta Neurochir (Wien) 147:1121-1130, 2005

24. Park HH, Oh MC, Kim EH, Kim CY, Kim SH, Lee KS, et al: Use of optical coherence tomography to predict visual outcome in parachiasmal meningioma. J Neurosurg 123:14891499,2015

25. Park W, Hong SD, Nam DH, Kong DS, Ryu G, Kim HY, et al: Nasoseptal flap elevation in patients with history of septal surgery: does it increase flap failure or cerebrospinal fluid leakage? World Neurosurg 93:164-167, 2016

26. Sade B, Lee JH: High incidence of optic canal involvement in tuberculum sellae meningiomas: rationale for aggressive skull base approach. Surg Neurol 72:118-123, 2009

27. Schick U, Hassler W: Surgical management of tuberculum sellae meningiomas: involvement of the optic canal and visual outcome. J Neurol Neurosurg Psychiatry 76:977-983, 2005

28. Seol HJ, Park HY, Nam DH, Kong DS, Lee JI, Kim JH, et al: Clinical outcomes of tuberculum sellae meningiomas focusing on reversibility of postoperative visual function. Acta Neurochir (Wien) 155:25-31, 2013

29. Song SW, Kim YH, Kim JW, Park CK, Kim JE, Kim DG, et al: Outcomes after transcranial and endoscopic endonasal approach for tuberculum meningiomas - a retrospective comparison. World Neurosurg 109:e434-e445, 2018

30. Taha AN, Erkmen K, Dunn IF, Pravdenkova S, Al-Mefty O:
Meningiomas involving the optic canal: pattern of involvement and implications for surgical technique. Neurosurg Focus 30(5):E12, 2011

31. Zada G, Fredrickson VL, Wrobel BB: Extended endoscopic endonasal approach for resection of tuberculum sellae meningioma. Neurosurg Focus 43 (VideoSuppl2):V2, 2017

32. Zevgaridis D, Medele RJ, Müller A, Hischa AC, Steiger HJ: Meningiomas of the sellar region presenting with visual impairment: impact of various prognostic factors on surgical outcome in 62 patients. Acta Neurochir (Wien) 143:471476, 2001

\section{Disclosures}

The authors report no conflict of interest concerning the materials or methods used in this study or the findings specified in this paper.

\section{Author Contributions}

Conception and design: YH Kim, Kong, CK Hong, SD Hong, DG Kim. Acquisition of data: all authors. Analysis and interpretation of data: YH Kim. Drafting the article: YH Kim, Kong, CK Hong.

\section{Correspondence}

Yong Hwy Kim: Seoul National University Hospital, Seoul, Republic of Korea.kimyh96@snu.ac.kr. 